\title{
Sparsity-inducing Dictionaries for Effective Action Classification
}

\author{
Debaditya Roy*, M. Srinivas*, C. Krishna Mohan* \\ Visual Learning and Intelligence Group (VIGIL) \\ Department of Computer Science and Engineering \\ Indian Institute of Technology Hyderabad
}

Kandi, Hyderabad (India) - 502285

\begin{abstract}
Action recognition in unconstrained videos is one of the most important challenges in computer vision. In this paper, we propose sparsity-inducing dictionaries as an effective representation for action classification in videos. We demonstrate that features obtained from sparsity based representation provide discriminative information useful for classification of action videos into various action classes. We show that the constructed dictionaries are distinct for a large number of action classes resulting in a significant improvement in classification accuracy on the HMDB51 dataset. We further demonstrate the efficacy of dictionaries and sparsity based classification on other large action video datasets like UCF50.
\end{abstract}

Keywords: Action Classification, Dictionary Learning, Sparse Representation, Action Bank features

\section{Introduction}

Action recognition is the process of extracting human action patterns from real video streams. It can be used in diverse applications like automated video indexing of huge on-line video repositories like Youtube \& Vimeo, analysing video surveillance systems in public places, humancomputer interaction, sports analysis etc. Actions are defined as single-person activities like "walking", "waving", "punching" etc. If the action video contains only one distinct human action, the task is to classify the video into one of the different categories. It has been shown in [1] that both spatial and temporal information are important for action representation. However, features which are shared across action classes are not suitable to build discriminative dictionaries. For example, "running" is a part of both "cricket bowling" and "soccer penalty". In such a case, the main action (bowling / penalty taking) occupies a small fraction of the entire duration of the video. Hence, it is difficult with just spatio-temporal descriptors to classify such actions with high credibility. Action bank [2] captures the similarity of the video with the class it belongs

\footnotetext{
${ }^{*}$ Corresponding author

Email addresses: cs13p1001@iith.ac. in (Debaditya Roy), cs10p002@iith.ac.in (M. Srinivas), ckm@iith.ac.in (C. Krishna Mohan)
} 
to and dissimilarity with other classes. Since, running occurs before bowling(or penalty taking), this temporal dependence can be exploited to produce a more unique representation for "cricket bowling" (or soccer penalty) which is useful for classification.

In this work, we construct sparsity-inducing dictionaries built specifically for action classification. Such a sparse dictionary based representation highlights discriminative information about various action classes. Also, these dictionaries distinctly represent the different action classes of HMDB51 dataset. Since dictionary learning has no strict convergence criteria, the dictionaries are trained until reasonable classification performance is obtained. On the HMDB51 dataset which contains many diverse and challenging views of human actions, dictionaries achieve very low mis-classification rate.

The rest of the paper is organized as follows. In section 2 we provide an overview of the various feature descriptors and sparsity based methods which have been applied for action classification. In section 3, we present the proposed sparsity based classification scheme in detail. In section 4 , we describe the performance of the proposed approach on two large action datasets - UCF50 and HMDB51. Finally, section 5 gives the conclusion for this work.

\section{Related Work and Analysis}

The challenges in action recognition have been studied with great interest in the computer vision community. Schuldt et al. [3] introduced the KTH [4] dataset which consists of six action categories. A support vector machine (SVM) was used for classfication with local space-time features. In [5], Kläser et al. presented the histogram of oriented 3D spatio-temporal gradients which is essentially a collection of quantized 2D histograms collected from each frame of the video. Kuehne et al. [6] introduced the HMDB51 dataset [7] for action recognition. Features such as histogram of oriented gradients (HOG), histogram of optical flow (HOF) and C2 were extracted and then a radial basis SVM was used for classification. Kliper et al. [8] proposed the use of motion interchange patterns i.e the change of one motion leading to another to describe a distinct action.

Solmaz et al. [9] presented the idea of gist, a global video descriptor which essentially computes the 3-D discrete Fourier transform of a given video clip using 68 3-D Gabor filters placed in 37 and 31 orientations. A trajectory based local descriptor TrajMF was proposed by Jiang et al. [10] which works on top of local feature descriptors like HOG, HOF etc. and captures global and local reference points to characterize motion information. Wang et al. [1] employed the idea of dense trajectories by estimating human motion, accurate camera motion estimation and removing inconsistent matches. In [11], Wu et al. denoted each action class as an event and assigned a latent variable to it. The crucial motion patterns in each event were then captured using latent models. These latent models were then used to construct latent structural SVMs, max-margin hidden conditional random fields and latent SVMs. Using a latent spatio-temporal compositional model in [12], actions were simplified in terms of spatio-temporal And-Or Graphs.

Recent works like [13] and [14] indicate that self-learned features can be as competitive as manually generated features for action classification. These works focus on convolutional neural networks $(\mathrm{CNN})$ and $\mathrm{CNN}$-based recurrent neural networks (RNN). In [13], consecutive frames 
of a video were processed through separate CNNs and then the outputs are fused in various configurations to obtain the best possible discriminative representation. $\mathrm{Ng}$ et al. [14] combined the outputs of CNNs from 15 or more subsequent frames into a RNN using long short term memory units (LSTM) to obtain a temporal representation. The performance was slighty better than improved dense trajectory features on the UCF101 dataset. A deep parsing based CNN network was proposed in [15] to build an end-to-end relation between the input human image and the structured outputs for human parsing. In [16], images representing humans actions are classified and localized using multiple regions for training a region-based CNN (R-CNN). Lin et al.[17] developed a deep structural model for 3D action recognition. Traditional CNNs were fused with a latent temporal model for representing temporal variation. Regularization was introduced in the form of radius-margin bound for better generalization. A similar architecture is presented in [18]. In [19], handcrafted features were augmented with CNN outputs learnt from various input sources using multiplicative fusion to classify actions. From the literature it can be seen that CNNs can provide a good representation of human actions.

Action bank features are useful for semantic representation of videos proposed by Sadanand and Corso [2]. This representation of videos is achieved by applying 73 spatio-temporal volume detectors on a video clip. There are 205 action templates having an average spatial resolution of approximately $50 \times 120$ pixels and a temporal length of $40-50$ frames. This contributes to a 14965-dimensional feature vector for each video clip under consideration. The templates perform classification by detection and give a global description of videos. Action bank produces a single feature vector for an entire video clip which is larger $(14965 \times 1)$ as compared to the number of video clips per class in any of the standard datasets $(\approx 100)$. The resultant matrix is a "fat" matrix $(14965 \times 100)$ which gives rise to an under-complete dictionary learning setting. In this work, we explore sparsity-inducing dictionaries to achieve a discriminative representation of human actions.

Dictionaries have been previously used in literature for action classification. In [20], information maximization was used for building discriminative dictionaries. These dictionaries were used to represent action attributes to classify images representing human actions. Sparse modeling for motion analysis was proposed by Castrodad et al. [21]. Using highly redundant features, a two-level pipeline was built to distinguish human actions. An evaluation of three different dictionary types - shared, class-specific and concatenated for the KTH, Weizmann and Hollywood2 datasets was done in [22]. The study found that the class-specific dictionaries perform better on an average than the shared and concatenated types. In [23], a sparse dictionary was constructed in an on-line manner for each incoming frame. In case of normal activity, consequent frames are related to each other and dictionary update is minimal. However, any abnormal activity would cause a major change in the dictionary. A new descriptor known as locally weighted word context was introduced in [24] which is a context-aware spatio-temporal descriptor. A sparse dictionary based on the descriptor was constructed using the joint $\ell_{2,1}$-norm where each action category share similar atoms in the dictionary.

In [25], feature encoding methods like vector quantization (VQ), Fisher vector (FV), localityconstrained linear coding (LLC) and soft assignment (SA) were evaluated in the context of sparse coding. Fisher vector was found to be the most suitable representation to forms sparse dictionaries using improved dense trajectory (IDT) features [1] on HMDB51 and UCF101 datasets. Lu et al. [26] proposed a new sparse coding scheme in which optimized local pooling was used 
to form discriminative dictionaries. A multilevel branch-and-bound approach was developed to achieve action localization on videos. This extensive review of sparsity-based dictionary learning methods for action recognition showed that dictionaries can be effectively used for action classification. In [27], the dictionary learning phase and feature encoding phase (e.g. fisher vector with GMM) were studied separately for action recognition. Various features like spatio-temporal interest points (STIP), cuboids and IDT were used to construct discriminative dictionaries. These dictionaries were formed using GMM, $k$-means, orthogonal matching pursuit and sparse coding. They found that the efficacy of dictionaries was not dependant on different feature encoding techniques. In [28], the authors proposed a representation for action recognition based on high-order statistics of the interaction among regions of interest in actions called action-gons. These actiongons were extracted using IDT features and served as discriminative dictionaries. Hence, it can be observed from the literature that dictionaries are able to provide a robust representation of actions on different kinds of features.

\section{Sparsity-inducing dictionaries for action classification}

In this section, a detailed discussion of the proposed method is presented. The classification scheme in typical dictionary learning consists of two phases - dictionary construction from training examples (training) and sparsity based evaluation of test clip (testing). The detailed block diagram of the entire approach is given in figure 1. In the training phase, dictionaries are constructed for each class using online dictionary learning (ODL) and then concatenated to form a single dictionary. Testing phase comprises of computing the sparsity of a test clip with the concatenated dictionary based on the $\ell_{1}$-norm. The class assigned to the video is the one having largest $\ell_{1}$-norm for the given test clip.

\subsection{Dictionary based representation}

The aim of dictionary learning is to represent dense features in form of a representative dictionary. This dictionary induces a sparse notation for the dense feature while retaining the information contained in the feature. Given a set of $m$-dimensional features $\left\{\mathbf{x}_{i}\right\}_{i=1}^{n}$, the $K$-SVD based dictionary learning method [29] finds an optimal dictionary $\mathbf{D}_{m \times k}$ and a sparse matrix $\boldsymbol{\Phi}_{k \times n}$ which best represent the features, as follows:

$$
\arg \min _{\mathbf{D}, \boldsymbol{\Phi}}\|\mathbf{V}-\mathbf{D} \boldsymbol{\Phi}\|_{F}^{2}
$$

subject to

$$
\left\|\phi_{i}\right\|_{0} \leq T_{0} \forall i
$$

where $\phi_{i}$ represents $i^{\text {th }}$ column of the sparse matrix $\mathbf{\Phi}, \mathbf{X}$ is the matrix whose columns are $\mathbf{x}_{i}$, and $T_{0}$ is the sparsity parameter. Here, $\|\mathbf{A}\|_{F}$ denotes the Fröbenius norm which is defined as $\|\mathbf{A}\|_{F}=\sqrt{\sum_{i j} \mathbf{A}^{\mathbf{2}}{ }_{i j}}$. The $K$-SVD algorithm alternates between sparse coding (finding $\boldsymbol{\Phi}$ ) and dictionary update (finding $\mathbf{D}$ ) steps.

On-line dictionary learning (ODL) is an on-line version of $k$-SVD algorithm proposed by Mairal et al. [30]. The sparse stage in ODL is a Cholesky-based implementation of LARS-lasso 


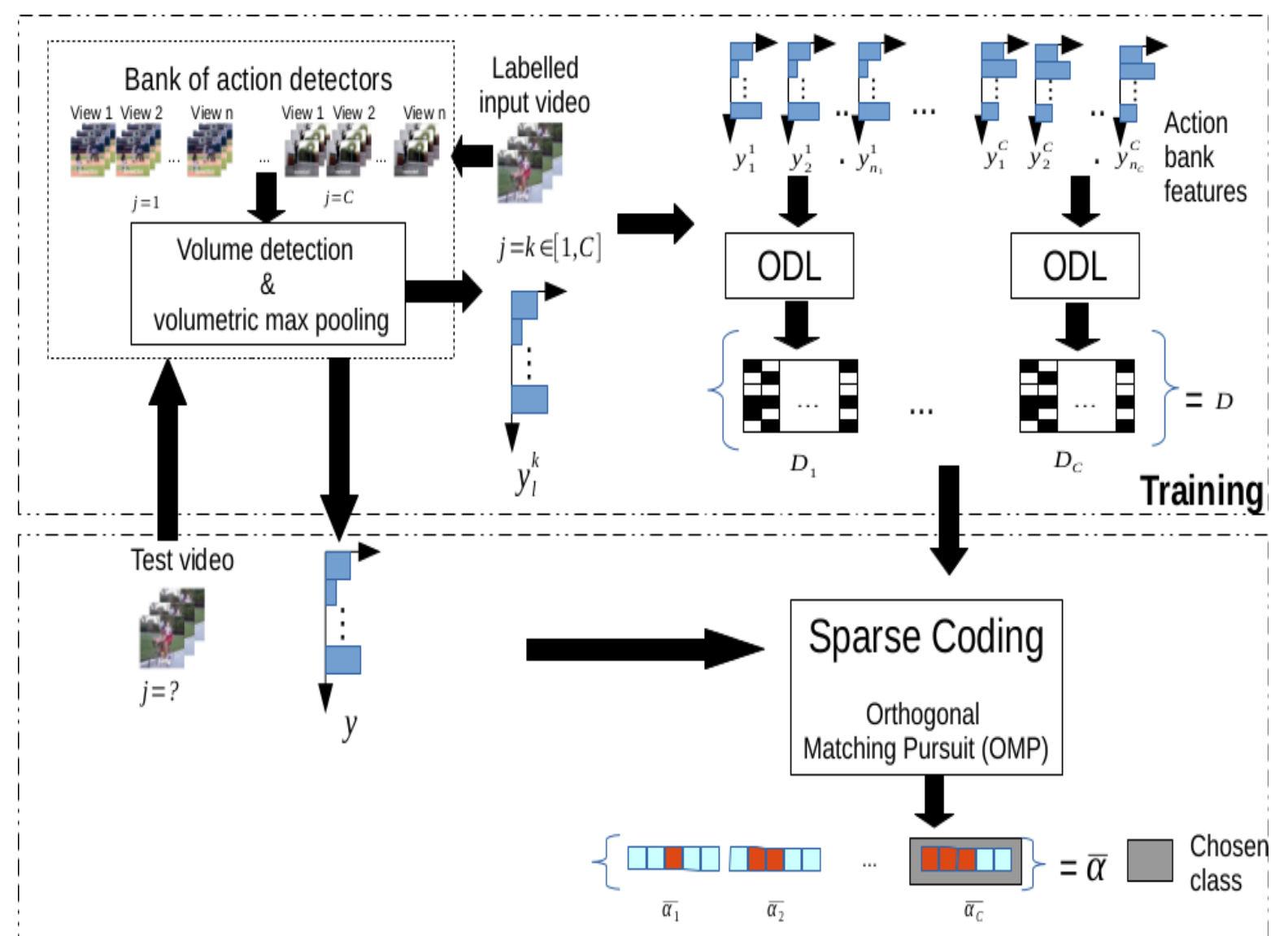

Testing

Figure 1: Flowchart of the proposed approach

algorithm which is similar to $k$-SVD (equation 1) but with a different sparsity constraint based on the $\ell_{1}$-norm of $\phi$ as given in equation 3. The sparse vector for the $t^{\text {th }}$ incoming feature, $\phi_{t}$ is found using the optimization function :

$$
\arg \min _{\mathbf{D}, \Phi}\|\mathbf{V}-\mathbf{D} \Phi\|_{2}^{2}+\lambda\left\|\phi_{\mathbf{t}}\right\|_{1}
$$

In the dictionary update stage, to avoid tuning the learning rate, block coordinate descent is used. It learns one example at a time giving the on-line nature similar to on-line stochastic approximation algorithms. This feature is particularly useful for large datasets. The dictionary $\mathbf{D}_{t}$ after incorporating the $t^{\text {th }}$ example, is calculated with respect to the previous dictionary $\mathbf{D}_{t-1}$ as :

$$
\arg \min _{\mathbf{D} \in C} \frac{1}{t} \sum_{i=1}^{t} \frac{1}{2}\left\|\mathbf{V}-\mathbf{D}_{t-1} \boldsymbol{\Phi}_{t-1}\right\|_{2}^{2}+\lambda\left\|\phi_{i}\right\|_{1},
$$

where $C$ determines the action classes to be trained for. 


\subsection{Sparsity based classification}

Suppose we have $N$ classes, $C_{1}, C_{2}, \ldots, C_{N}$ consisting of $K_{1}, K_{2}, \ldots, K_{N}$ number of training features, respectively. The features belonging to the same class $C_{i}$ lie approximately close to each other in a low-dimensional subspace [31]. Let $\mathbf{b}$ be a input feature belonging to the $p^{\text {th }}$ class, then it is represented as a linear combination of the training samples belonging to class $p$ :

$$
\mathbf{b}=\mathbf{D}_{\mathbf{p}} \phi_{\mathbf{p}},
$$

where $\mathbf{D}_{\mathbf{p}}$ is a $m \times K_{p}$ dictionary whose columns are the training samples in the $p^{\text {th }}$ class and $\phi_{p}$ is a sparse vector for the same class.

In the classification process, the sparse vector $\phi_{j}$ is found for the test feature $\mathbf{b}_{j}$ using the dictionaries of training samples $\mathbf{D}=\left[\mathbf{D}_{1}, \ldots, \mathbf{D}_{N}\right]$ by solving the following optimization problem:

$$
\arg \min _{\phi} \frac{1}{2}\left\|\mathbf{b}_{j}-\mathbf{D} \phi_{j}\right\|_{2}^{2}
$$

subject to

$$
\left\|\phi_{j}\right\|_{1} \leq T
$$

and

$$
\hat{i}=\arg \max _{i}\left\|\delta_{i}\left(\phi_{j}\right)\right\|_{1}, i=1, \cdots, N
$$

where $\delta_{i}$ is a characteristic function that selects the coefficients for class $C_{i}, T$ represents the sparsity threshold. A test clip $\mathbf{b}_{j}$ is assigned to class $C_{i}$ if the absolute sum of sparsity coefficients associated with the $i^{t h}$ dictionary is maximum among other classes. This criteria was chosen instead of counting the number of non-zero coefficients as it was found to be better at classification. The reason for using sparsity as classification is that while forming a dictionary for a class, we admit the sparsest representation of features belonging to that class. So, if a test feature belongs to a certain class, it should ideally admit the sparsest representation with respect to that class dictionary and no other.

\section{Results and Evaluation}

In this section, a critical evaluation of the proposed method is presented. The main goal is to establish the robustness of sparse representation on large datasets like HMDB51 and UCF50. Further evaluation is done to determine the optimal dictionary size with respect to classification accuracy.

\subsection{HMDB51}

The HMDB51 dataset is a very large human action dataset containing 51 action categories, with at least 101 clips for each category. The dataset includes a total of 6,766 video clips extracted from movies, the Prelinger archive, Youtube and Google videos. Such a variety of sources which have contributed to this database make it very realistic and challenging. Three distinct training and testing splits have been selected from the dataset as provided in [7], with 70 training and 30 testing clips for each category. Some of the sample actions are shown in figure 2 . 

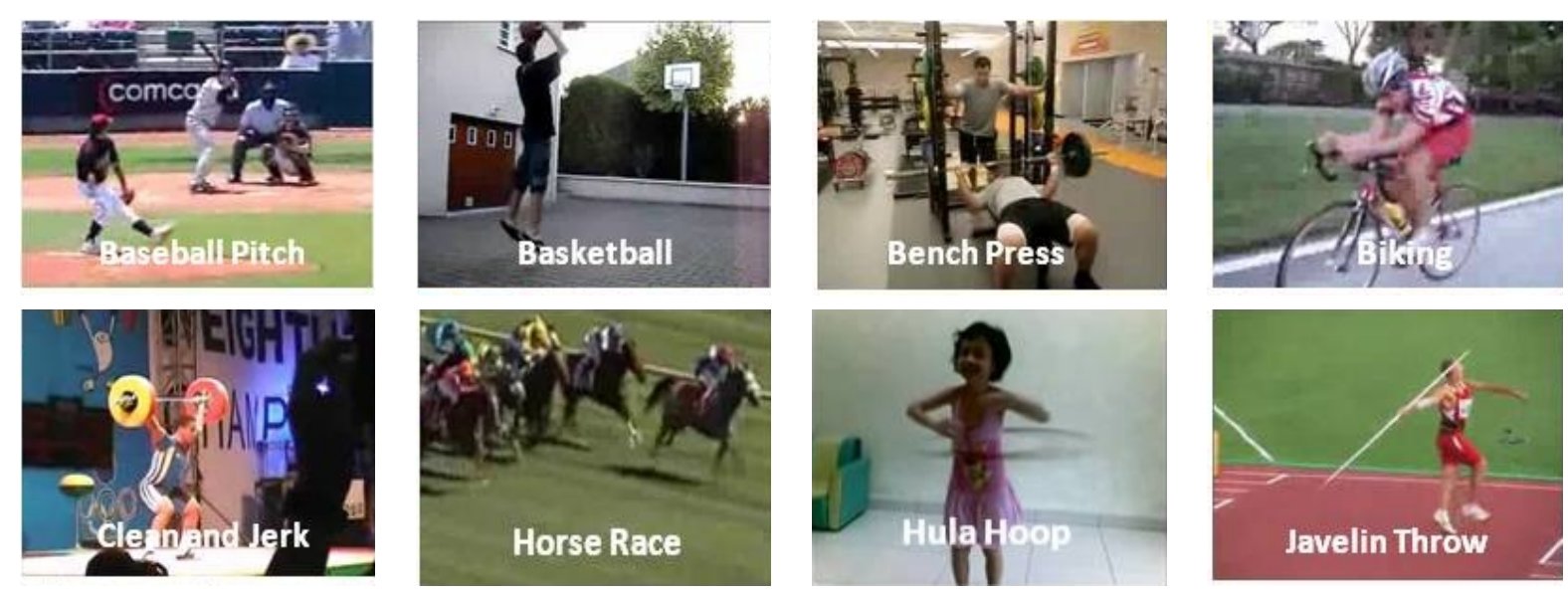

Figure 2: Sample actions from HMDB51 dataset

\section{2. $U C F 50$}

The UCF50 dataset was introduced in [32], consists of 50 sport action categories and all the videos denoting the actions were collected from YouTube. The dataset consists of more than 100 video clips for each category and gives plenty of variety in terms of camera motion, object appearance and pose, object scale, viewpoint, cluttered background, illumination conditions, etc. The official train/test splits are available at [33] and were used in this paper to maintain comparability with the previous literature on these datasets.

\subsection{Performance Evaluation}

A summary of the classification performance of previous approaches in literature applied on HMDB51 is presented in Table 1. It can be observed from the table that single frame based features like HOG/HOF[6], C2[6] , motion interchange patterns [8] demonstrate high mis-classification as they do not consider temporal context while describing action. On the other hand, trajectory features [11], [1], [28] which consider multiple frames to provide temporal description of the motion perform better than single frame based features. Action bank is also one such representation which uses a spatio-temporal volume across multiple frames but performs slightly better than single frame based features. However, representing action bank features in terms of sparsityinducing dictionaries improves the performance significantly as shown in table 1 . It can be noticed that a similar dictionary transformation of improved dense trajectory features [27] betters the performance only slightly (57.2 to 59.7\%). This shows the suitability of action bank features for sparse dictionary based representation. Further, it is also evident from table 1 that the proposed method demonstrates significantly higher classification accuracy than CNN and CNN based RNN networks presented in [19], [39], [37], [34] and [38].

Also, we conducted experiments with 3D-scale invariant feature transform (SIFT) features [42] for learning sparse dictionaries. Table 2 presents a comparison of classification performance among various features used for learning dictionaries on the HMDB51 dataset. As reported in table 2 . the best classification performance of $22.08 \%$ was obtained for 3D-SIFT features with a dictionary of size 80. Other features previously used for building dictionaries include IDT features[27] 
Table 1: Comparison of classification performance on the HMDB51 action dataset

\begin{tabular}{|c|c|c|}
\hline Method & Feature & Accuracy $(\%)$ \\
\hline \multicolumn{3}{|c|}{ Single-frame based feature } \\
\hline Kuehne et al. [6] & $\mathrm{HOG} / \mathrm{HOF}$ & 20.20 \\
\hline Kuehne et al. [6] & $\mathrm{C} 2$ & 23.18 \\
\hline Kliper-Gross et al. [8] & Motion Interchange Patterns & 29.17 \\
\hline \multicolumn{3}{|c|}{ Multiple-frame based feature } \\
\hline Solmaz et al. [9] & $\begin{array}{c}\text { Frequency based } \\
\text { 3D spatio-temporal features }\end{array}$ & 29.20 \\
\hline Jiang et al. [10] & $\begin{array}{l}\text { Trajectory on motion } \\
\text { reference points }\end{array}$ & 40.70 \\
\hline Srivastava et al. [34] & RNN with LSTM & 44.1 \\
\hline Wang et al. [1] & Dense trajectory & 44.75 \\
\hline Wu et al. [11] & Dense trajectory-aligned & 49.46 \\
\hline Liu et al. [35] & Multiple features & 49.95 \\
\hline Lan et al. [36] & Local handcrafted features & 52.4 \\
\hline Park et al. [37] & Multiple CNNs & 54.9 \\
\hline Wang et al. [1] & IDT & 57.20 \\
\hline Wang et al. [28] & $\begin{array}{c}\text { Action-gons }+ \\
\text { Sparse Dictionaries }\end{array}$ & 58 \\
\hline Sun et al. [19] & Factorized Spatio-Temporal CNNs & 59.1 \\
\hline Simonyan et al. [38] & Two stream CNNs & 59.4 \\
\hline Wang et al. [39] & $\begin{array}{c}\text { Temporal Pyramid Pooling } \\
\text { based CNN }\end{array}$ & 59.7 \\
\hline Peng et al. [27] & IDT + Sparse Dictionaries & 59.7 \\
\hline Lan et al. [40] & Space-time Extended Descriptor & 62.1 \\
\hline Lan et al. [41] & Long short term motion & 63.7 \\
\hline Sadanand et al. [2] & Action bank & 26.90 \\
\hline Proposed approach & $\begin{array}{c}\text { Action bank + } \\
\text { Sparse Dictionaries }\end{array}$ & 99.87 \\
\hline
\end{tabular}

and action-gons[28]. All these representations are based on spatio-temporal interest points but yield lower performance than action bank. This shows that features that highlight similarities or dissimilarities across classes enhances the dictionary representation providing higher classification performance.

Dictionaries constructed for sample classes of HMDB51 and UCF50 are presented in figures 3 \& 4, respectively. The variability in actions of HMDB51 in terms of body movement, posture and overall appearance is adequately captured in the dictionaries. It is clearly evident that the dictionaries formed for the classes of HMDB51 are indeed distinct from one another. This il- 
Table 2: Performance comparison of sparsity-based dictionaries using different features on the HMDB51 action dataset

\begin{tabular}{|c|c|}
\hline Feature & Accuracy (\%) \\
\hline 3D-SIFT & 22.08 \\
\hline Action-gons [28] & 58 \\
\hline Improved dense trajectory [27] & 59.7 \\
\hline Action Bank & $\mathbf{9 9 . 8 7}$ \\
\hline
\end{tabular}

lustrates that features belonging to different classes do not share a sparse neighbourhood. These distinct dictionaries contribute to better classification performance of dictionaries on the HMDB51 dataset. On the other hand, the dictionaries constructed for few of the classes of UCF50 bear strong similarities. The dictionaries corresponding to classes such as "javelin throw", "jumping jack", "kayaking", "playing guitar", "nunchunks", "pole vault", "pull ups" and "volleyball spiking" are quite similar making it hard to discriminate these classes with sparsity-inducing dictionaries which contributes to lower classification performance on the UCF50 dataset as can be seen in table 3 .

In figure 5, the confusion matrix of the UCF50 dataset is presented. "Pole vault" is misclassified as "kayaking" and "biking" is misclassified as juggling balls. Similarly, "walking with dog" is confused to be "tennis swing". These confusions are due to the fact that their representative dictionaries are almost identical as shown in figure 4. The results presented here are an extension to the work presented in [43].

In table 3, we present the performance of the proposed method on the UCF50 dataset. It can be see the dictionaries constructed from action bank features perform reasonably well as compared to state-of-the-art but not as well as action bank features. This shows that original features are more discriminative than the sparsity-inducing dictionaries. Further, it also illustrates that applying sparsity constraints while constructing dictionaries may not always lead to better discriminative representation.

Table 3: Classification performance on the UCF50 dataset

\begin{tabular}{|c|c|}
\hline Method & Accuracy (\%) \\
\hline Kliper-Gross et al. [8] & 72.60 \\
\hline Solmaz et al. [9] & 73.70 \\
\hline Reddy and Shah [32] & 76.90 \\
\hline Todorovic et al. [44] & $\mathbf{8 1 . 0 3}$ \\
\hline Sadanand et al. [2] (Action bank) & 76.40 \\
\hline Proposed approach & 72.46 \\
\hline
\end{tabular}

\subsection{Classification Performance vs. Dictionary Size}

The primary objective of dictionary learning is reconstruction. However, over-fitted dictionaries with perfect reconstruction are not desirable as variability in test examples cannot be handled 

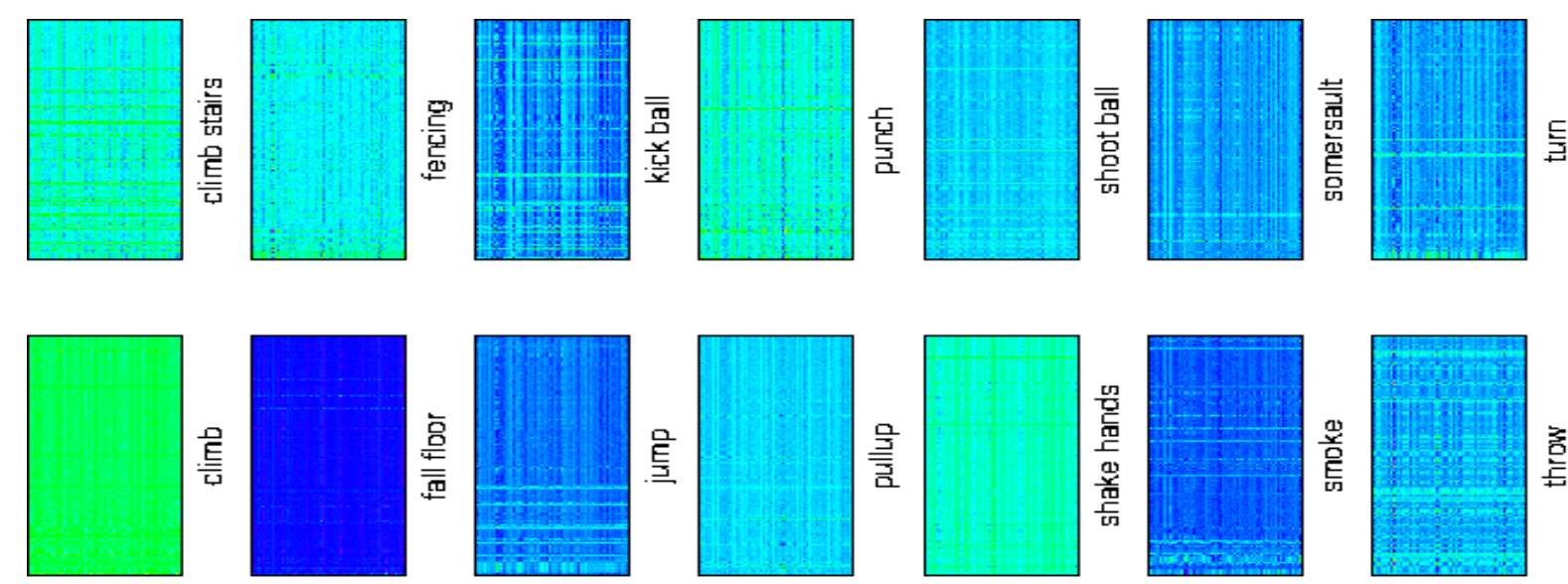

홀
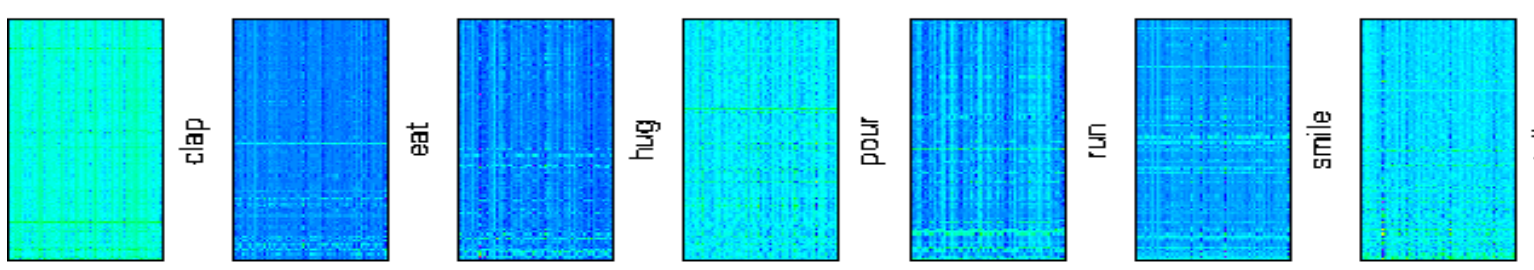

동
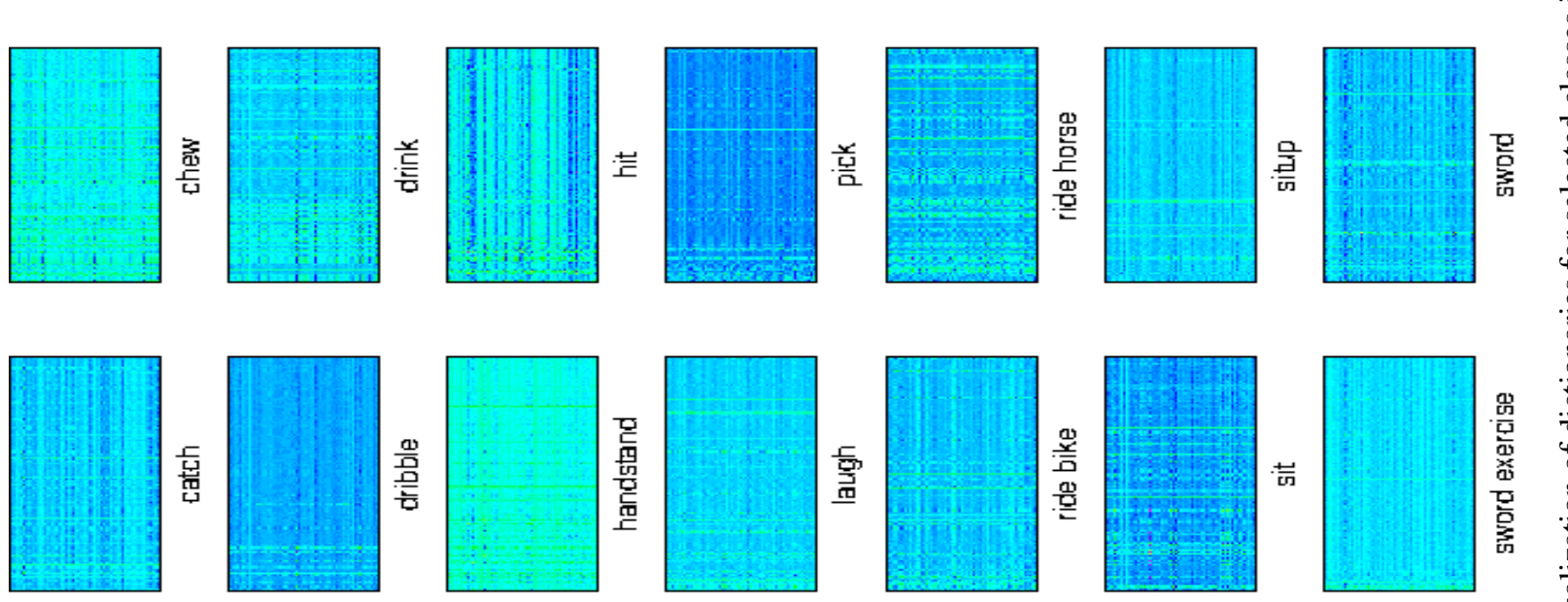

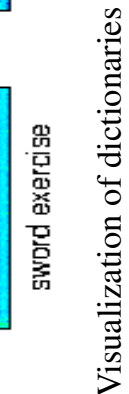
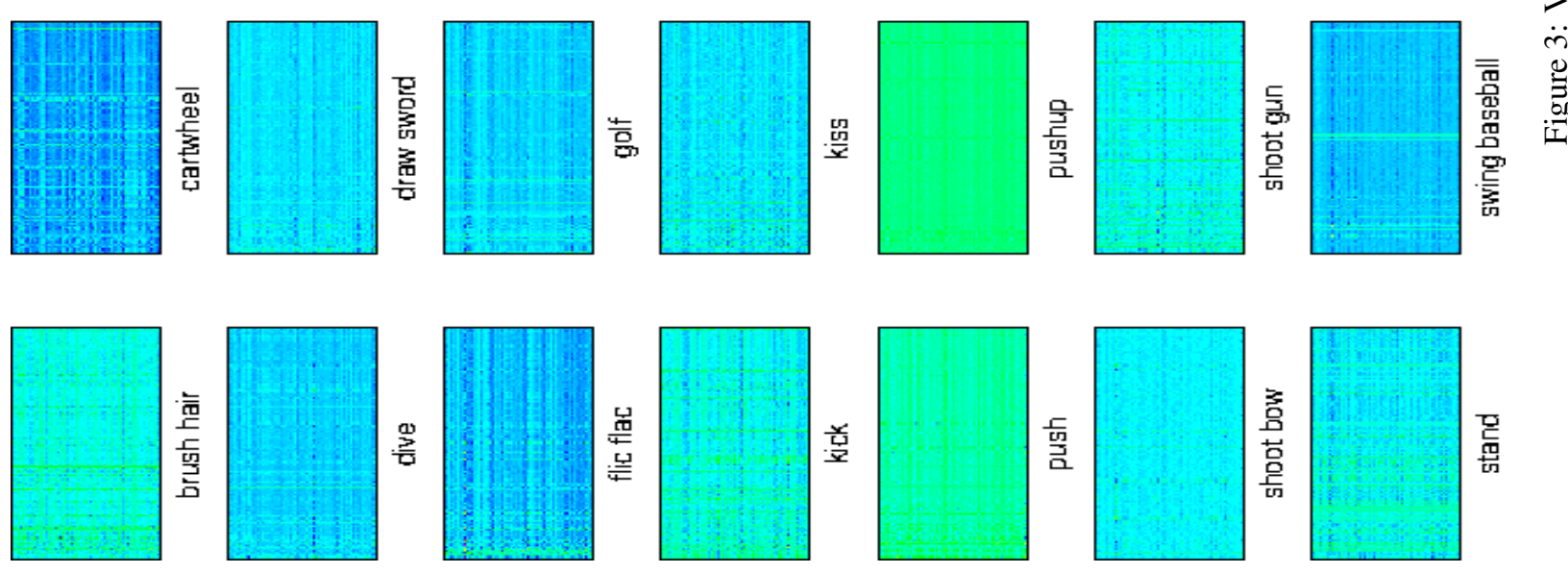

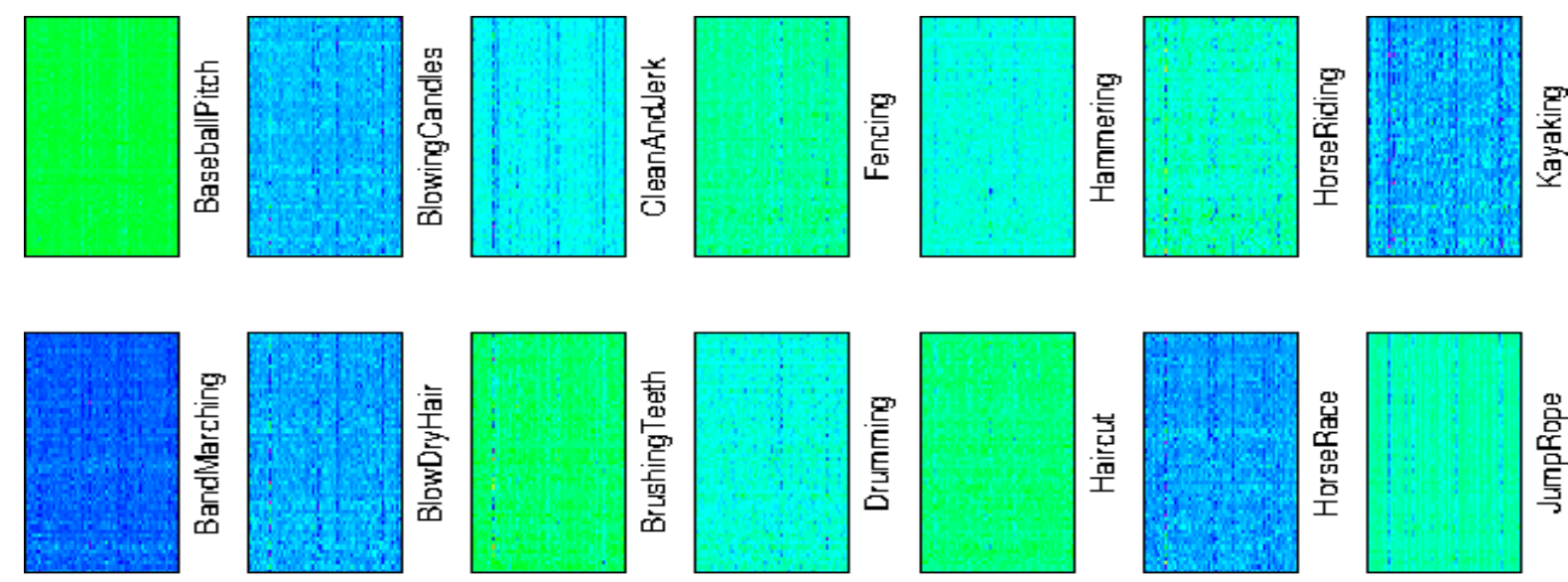

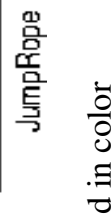
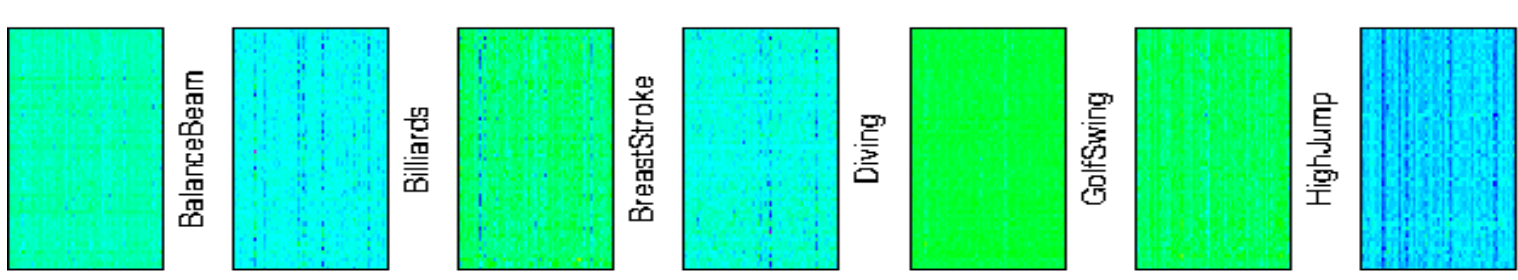

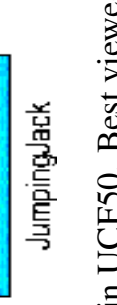
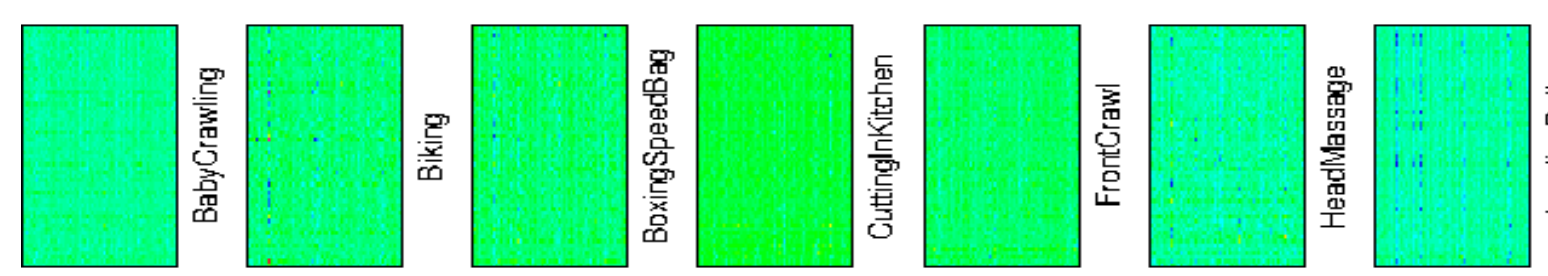

do
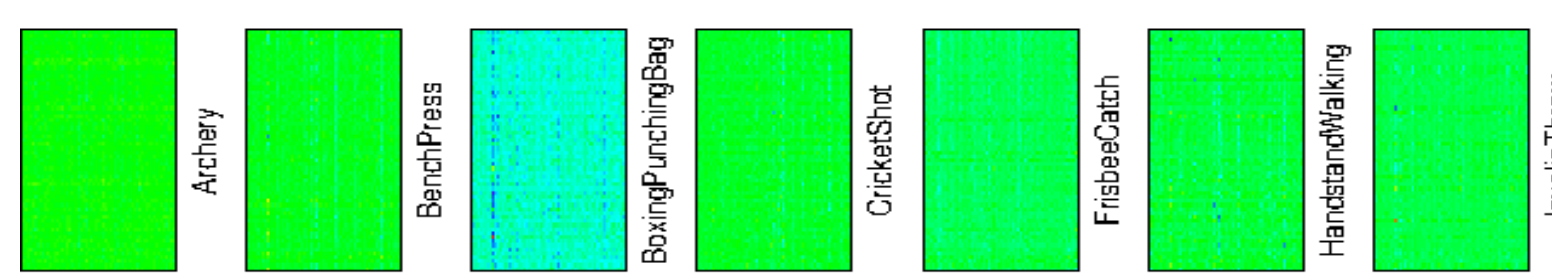

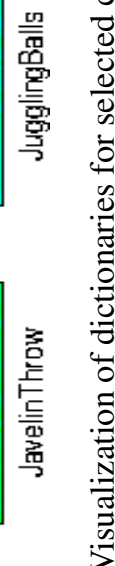
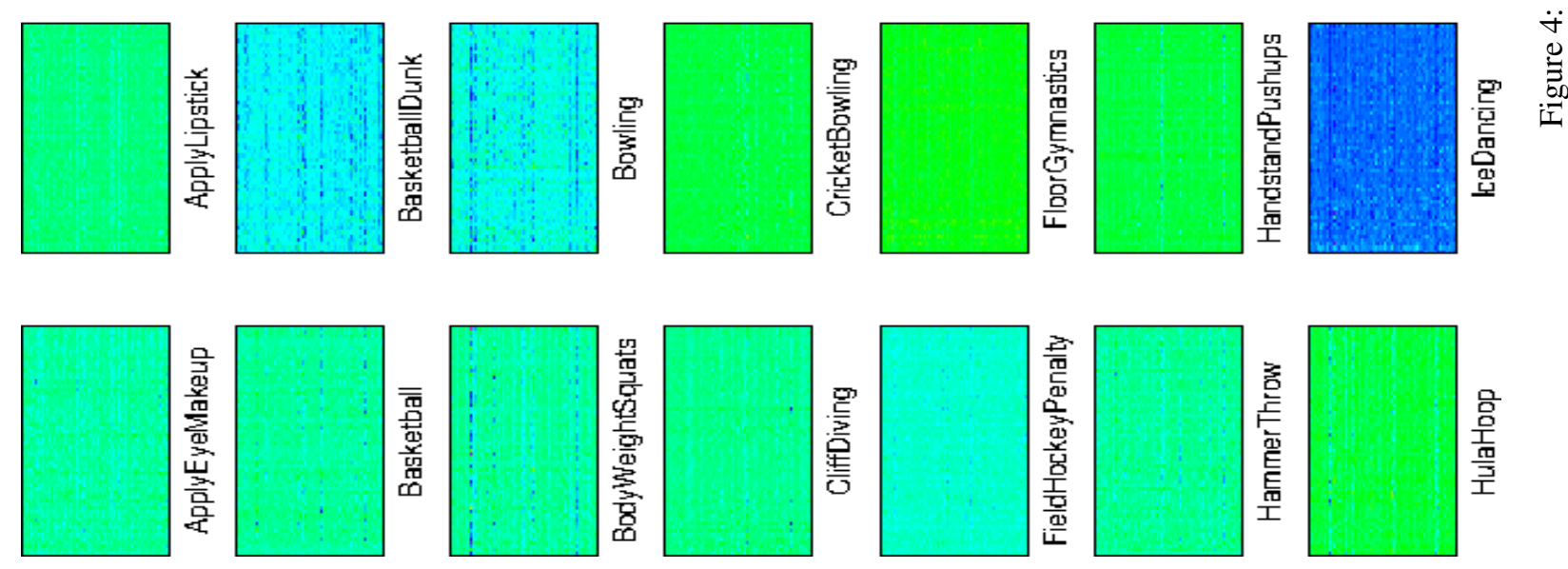


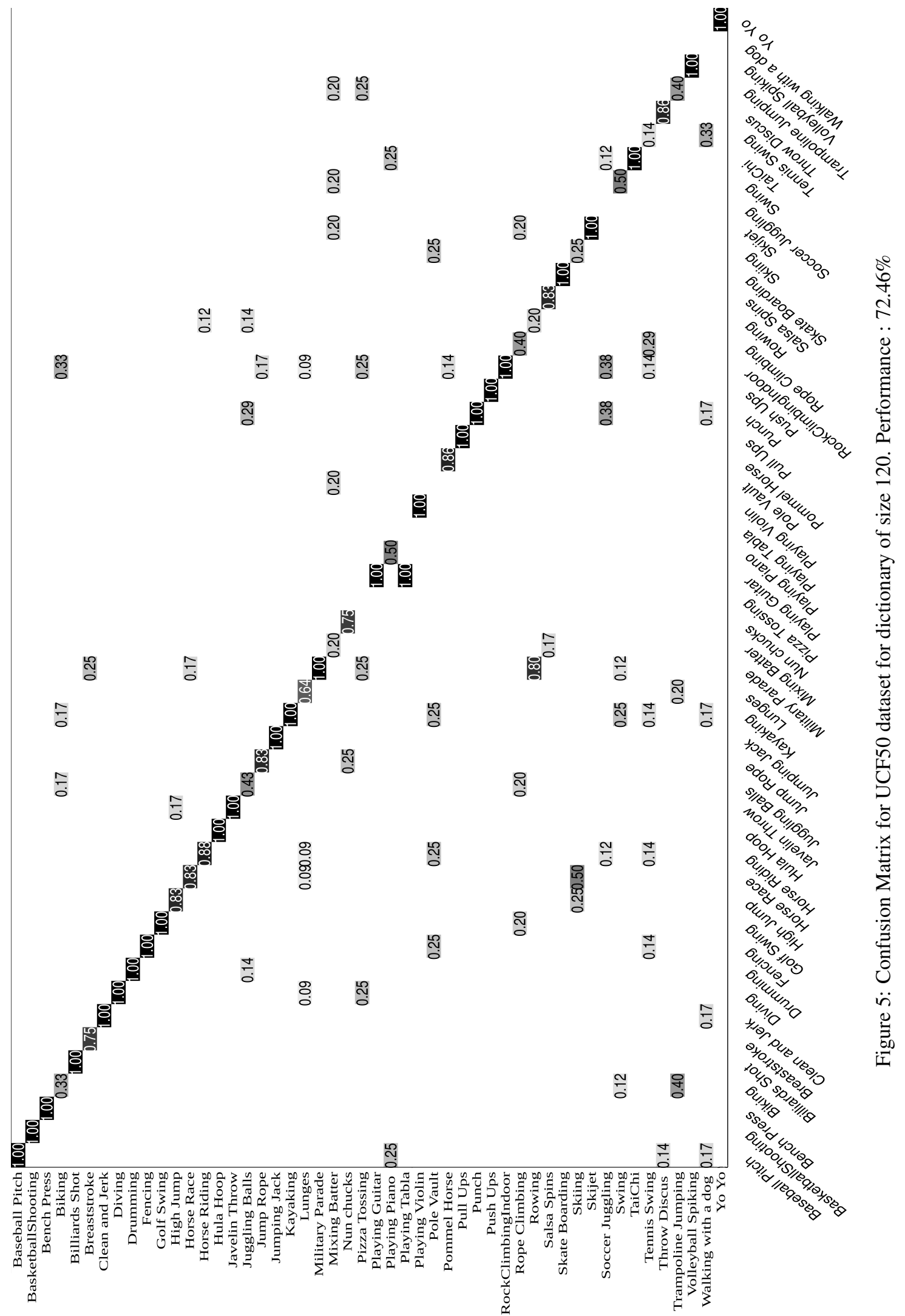


effectively leading to more mis-classification. Table 4 portrays the variation of recognition accuracy in terms of dictionary size for HMDB51 and UCF50 datasets. For HMDB51, the maximum performance is noted for dictionary size of 100 with sparsity (lambda value in SPAMS toolbox) set at 2, after which the performance degrades with increase in the dictionary size. In case of UCF50, best classification accuracy is obtained for dictionary size of 120 with sparsity set at 8 after which it degrades sharply. The reason could be that action bank features can be compressed with great effect till the point where all the discriminating characteristics remain. Beyond that point, increasing dictionary size leads to loss of information. This behavior is consistent across datasets and smaller dictionary sizes can produce a fair idea on the average overall classification performance. The only parameter to be tuned is sparsity. It also must be noted that optimal dictionary size is based on the objective at hand and the number of examples available for each class. In our case, the optimal dictionary size is reached where the reconstruction error is relatively low while maintaining high discrimination.

Table 4: Effect of dictionary size on performance (in \%)

\begin{tabular}{|c|l|l|}
\hline $\begin{array}{c}\text { Dictionary } \\
\text { Size }\end{array}$ & HMDB51 & UCF50 \\
\hline 60 & 92.33 & 51.6 \\
\hline 80 & 98.11 & 60 \\
\hline 100 & $\mathbf{9 9 . 8 7}$ & 63.9 \\
\hline 120 & 99.51 & $\mathbf{7 2 . 4 6}$ \\
\hline 140 & 98.23 & 69.6 \\
\hline 160 & 97.56 & 69.6 \\
\hline
\end{tabular}

\section{CONCLUSION}

The main goal of this work was to study dictionaries as an effective representation for action classification in videos. Sparse representation of multi-frame based features was exploited to obtain discriminative dictionaries. It was shown that these dictionaries distinctly represent the different action classes. Further, it was also shown that dictionaries learned from action bank features showed a four-fold improvement in classification accuracy over naïve action bank features on the HMDB51 dataset. However, we also found that class-wise dictionaries for some of the classes of UCF50 were similar causing mis-classification among examples of those classes. Future work would involve addressing the issue of classification among those classes whose dictionaries are found to be similar.

\section{ACKNOWLEDGEMENT}

We would like to thank Dr. Jason Corso for making the Action Bank features available for the UCF50 and HMDB51 datasets. We would also like to thank Dr. Julian Mairal for the SPAMS toolbox. 
[1] H. Wang, C. Schmid, Action recognition with improved trajectories, in: Computer Vision (ICCV). International Conference on, Sydney, Australia, 2013. URL http://hal.inria.fr/hal-00873267

[2] S. Sadanand, J. Corso, Action bank: A high-level representation of activity in video, in: Computer Vision and Pattern Recognition (CVPR). IEEE Conference on, 2012, pp. 1234-1241. doi:10.1109/CVPR.2012. 6247806

[3] C. Schuldt, I. Laptev, B. Caputo, Recognizing human actions: A local svm approach in: Proceedings of the Pattern Recognition (ICPR), 17th International Conference on Volume 3, IEEE Computer Society, Washington, DC, USA, 2004, pp. 32-36. doi:10.1109/ICPR.2004.747.

URL http://dx.doi.org/10.1109/ICPR.2004.747

[4] I. Laptev, B. Caputo,http://www.nada.kth.se/cvap/actions/

[5] A. Klaser, M. Marszalek, C. Schmid, A spatio-temporal descriptor based on 3d-gradients, in: British Machine Vision Conference (BMVC), 2008, pp. 275:1-10.

[6] H. Kuehne, H. Jhuang, E. Garrote, T. Poggio, T. Serre, HMDB: a large video database for human motion recognition, in: Computer Vision (ICCV), IEEE International Conference on, 2011, pp. 2556-2563.

[7] H. Jhuang, http://serre-lab.clps.brown.edu/resource/hmdb-a-large-human-motion-database.

[8] O. Kliper-Gross, Y. Gurovich, T. Hassner, L. Wolf, Motion interchange patterns for action recognition in unconstrained videos, in: Computer Vision (ECCV). European Conference on, 2012, pp. 425-438. URL http://www.openu.ac.il/home/hassner/projects/MIP

[9] B. Solmaz, S. Assari, M. Shah, Classifying web videos using a global video descriptor, Machine Vision and Applications 24 (7) (2013) 1473-1485. doi:10.1007/s00138-012-0449-x URL http://dx.doi.org/10.1007/s00138-012-0449-x

[10] Y.-G. Jiang, Q. Dai, X. Xue, W. Liu, C.-W. Ngo, Trajectory-based modeling of human actions with motion reference points, in: Computer Vision (ECCV). European Conference on, 2012.

[11] J. Wu, D. Hu, Learning effective event models to recognize a large number of human actions, Multimedia, IEEE Transactions on 16 (1) (2014) 147-158. doi:10.1109/TMM.2013.2283846.

[12] X. Liang, L. Lin, L. Cao, Learning latent spatio-temporal compositional model for human action recognition, in: Proceedings of the 21st ACM international conference on Multimedia, ACM, 2013, pp. 263-272.

[13] A. Karpathy, G. Toderici, S. Shetty, T. Leung, R. Sukthankar, L. Fei-Fei, Large-scale video classification with convolutional neural networks, in: CVPR, 2014.

[14] J. Y. Ng, M. J. Hausknecht, S. Vijayanarasimhan, O. Vinyals, R. Monga, G. Toderici, Beyond short snippets: Deep networks for video classification, CoRR abs/1503.08909.

URL http://arxiv.org/abs/1503.08909

[15] X. Liang, S. Liu, X. Shen, J. Yang, L. Liu, J. Dong, L. Lin, S. Yan, Deep human parsing with active template regression, Pattern Analysis and Machine Intelligence, IEEE Transactions on 37 (12) (2015) 2402-2414. doi: 10.1109/TPAMI.2015.2408360

[16] G. Gkioxari, R. B. Girshick, J. Malik, Contextual action recognition with r*cnn, CoRR abs/1505.01197. URL http://arxiv.org/abs/1505.01197

[17] L. Lin, K. Wang, W. Zuo, M. Wang, J. Luo, L. Zhang, A deep structured model with radius-margin bound for 3d human activity recognition, International Journal of Computer Vision (2015) 1-18doi:10.1007/ s11263-015-0876-z URL http://dx.doi.org/10.1007/s11263-015-0876-z

[18] K. Wang, X. Wang, L. Lin, M. Wang, W. Zuo, 3d human activity recognition with reconfigurable convolutional neural networks, in: Proceedings of the ACM International Conference on Multimedia, ACM, 2014, pp. 97-106.

[19] L. Sun, K. Jia, D.-Y. Yeung, B. E. Shi, Human action recognition using factorized spatio-temporal convolutional networks, in: Proceedings of the IEEE International Conference on Computer Vision, 2015, pp. 4597-4605.

[20] Q. Qiu, Z. Jiang, R. Chellappa, Sparse dictionary-based representation and recognition of action attributes, in: Computer Vision (ICCV), 2011 IEEE International Conference on, 2011, pp. 707-714. doi:10.1109/ICCV. 2011.6126307

[21] A. Castrodad, G. Sapiro, Sparse modeling of human actions from motion imagery, International Journal of Computer Vision 100 (1) (2012) 1-15. doi:10.1007/s11263-012-0534-7. 
URL http://dx.doi.org/10.1007/s11263-012-0534-7

[22] T. Guha, R. Ward, Learning sparse representations for human action recognition, Pattern Analysis and Machine Intelligence, IEEE Transactions on 34 (8) (2012) 1576-1588. doi:10.1109/TPAMI.2011.253.

[23] S. Han, R. Fu, S. Wang, X. Wu, Online adaptive dictionary learning and weighted sparse coding for abnormality detection, in: Image Processing (ICIP), 2013 20th IEEE International Conference on, 2013, pp. 151-155. doi : 10.1109/ICIP. 2013.6738032.

[24] H. Wang, C. Yuan, W. Hu, H. Ling, W. Yang, C. Sun, Action recognition using nonnegative action component representation and sparse basis selection, Image Processing, IEEE Transactions on 23 (2) (2014) 570-581. doi:10.1109/TIP.2013.2292550

[25] X. Peng, Q. Peng, Y. Qiao, J. Chen, M. Afzal, A study on unsupervised dictionary learning and feature encoding for action classification, CoRR abs/1309.0309. URL http://arxiv.org/abs/1309.0309

[26] S. Lu, J. Zhang, Z. Wang, D. D. Feng, Fast human action classification and \{VOI $\}$ localization with enhanced sparse coding, Journal of Visual Communication and Image Representation 24 (2) (2013) 127 - 136, sparse Representations for Image and Video Analysis. doi:http://dx.doi.org/10.1016/j.jvcir.2012.07. 008

URL http://www.sciencedirect.com/science/article/pii/S1047320312001253

[27] X. Peng, L. Wang, Y. Qiao, Q. Peng, A joint evaluation of dictionary learning and feature encoding for action recognition, in: Pattern Recognition (ICPR), 2014 22nd International Conference on, 2014, pp. 2607-2612. doi:10.1109/ICPR.2014.450.

[28] Y. Wang, B. Wang, Q. Dai, Y. Yu, Z. Tu, Action-gons: Action recognition with a discriminative dictionary of structured elements of varying granularity, ACCV, 2014.

[29] M. Aharon, M. Elad, A. Bruckstein, k -svd: An algorithm for designing overcomplete dictionaries for sparse representation, Signal Processing, IEEE Transactions on 54 (11) (2006) 4311-4322. doi : 10.1109/TSP. 2006. 881199

[30] J. Mairal, F. Bach, J. Ponce, G. Sapiro, Online dictionary learning for sparse coding, in: Proceedings of the 26th Annual International Conference on Machine Learning (ICML), ACM, New York, NY, USA, 2009, pp. 689-696. doi:10.1145/1553374.1553463

URL http://doi.acm.org/10.1145/1553374.1553463

[31] J. Wright, A. Yang, A. Ganesh, S. Sastry, Y. Ma, Robust face recognition via sparse representation, Pattern Analysis and Machine Intelligence, IEEE Transactions on 31 (2) (2009) 210-227. doi:10.1109/TPAMI. 2008.79 .

[32] K. K. Reddy, M. Shah, Recognizing 50 human action categories of web videos, Machine Vision and Applications 24 (5) (2013) 971-981. doi:10.1007/s00138-012-0450-4. URL http://dx.doi.org/10.1007/s00138-012-0450-4

[33] K. Soomro, A. R. Zamir, M. Shah, UCF101: A dataset of 101 human actions classes from videos in the wild, CoRR abs/1212.0402.

URL http://arxiv.org/abs/1212.0402

[34] N. Srivastava, E. Mansimov, R. Salakhutdinov, Unsupervised learning of video representations using lstms, CoRR abs/1502.04681. URL http://arxiv.org/abs/1502.04681

[35] J. Liu, Y. Huang, X. Peng, L. Wang, Multi-view descriptor mining via codeword net for action recognition, in: Image Processing (ICIP), 2015 IEEE International Conference on, 2015, pp. 793-797. doi:10.1109/ICIP. 2015.7350908.

[36] Z. Lan, S. Yu, M. Lin, B. Raj, A. G. Hauptmann, Handcrafted local features are convolutional neural networks, CoRR abs/1511.05045. URL http://arxiv.org/abs/1511.05045

[37] E. Park, X. Han, T. L. Berg, A. C. Berg, Combining multiple sources of knowledge in deep cnns for action recognition, in: IEEE Winter Annual Conference on Computer Vision, 2016.

[38] K. Simonyan, A. Zisserman, Two-stream convolutional networks for action recognition in videos, in: Advances in Neural Information Processing Systems, 2014, pp. 568-576. 
[39] P. Wang, Y. Cao, C. Shen, L. Liu, H. T. Shen, Temporal pyramid pooling based convolutional neural networks for action recognition, CoRR abs/1503.01224.

URL http://arxiv.org/abs/1503.01224

[40] Z. Lan, A. G. Hauptmann, Beyond spatial pyramid matching: Space-time extended descriptor for action recognition CoRR abs/1510.04565.

URL http://arxiv.org/abs/1510.04565

[41] Z. Lan, X. Li, M. Lin, A. G. Hauptmann, Long-short term motion feature for action classification and retrieval, CoRR abs/1502.04132.

URL http://arxiv.org/abs/1502.04132

[42] P. Scovanner, S. Ali, M. Shah, A 3-dimensional sift descriptor and its application to action recognition, in: Proceedings of the 15th international conference on Multimedia, ACM, 2007, pp. 357-360.

[43] D. Roy, M. Srinivas, C. K. Mohan, Sparsifying dense features for action classification in: Proceedings of the 2Nd International Conference on Perception and Machine Intelligence, PerMIn '15, ACM, New York, NY, USA, 2015, pp. 211-217. doi:10.1145/2708463.2709047.

URL http://doi.acm.org/10.1145/2708463.2709047

[44] S. Todorovic, Human activities as stochastic kronecker graphs, in: Computer Vision-ECCV 2012, Springer, 2012, pp. 130-143. 